\title{
Sterile neutrinos searches at IceCube
}

\section{Mattias Blennow}

Instituto de Física Teórica UAM/CSIC, Calle Nicolás Cabrera 13-15, Cantoblanco E-28049

Madrid, Spain

E-mail: m.blennowecsic.es

\section{Enrique Fernandez-Martinez}

Instituto de Física Teórica UAM/CSIC, Calle Nicolás Cabrera 13-15, Cantoblanco E-28049

Madrid, Spain

E-mail: enrique.fernandez-martinez@uam.es

\section{Julia Gehrlein}

Instituto de Física Teórica UAM/CSIC, Calle Nicolás Cabrera 13-15, Cantoblanco E-28049

Madrid, Spain

E-mail: julia.gehrlein@uam.es

\section{Josu Hernandez-Garcia*}

SISSA/INFN - Sezione di Trieste

E-mail: josu.hernandez@ts.infn.it

\section{Jordi Salvado}

Institut de Ciències del Cosmos, Universitat de Barcelona, Diagonal 647, E-08028 Barcelona, Spain

E-mail: jsalvado@icc.ub.edu

\begin{abstract}
Neutrino masses are one of the most promising open windows to physics beyond the Standard Model (SM). Several extensions of the SM which accommodate neutrino masses require the addition of right-handed neutrinos to its particle content. The capabilities of IceCube to search for sterile neutrinos with masses above $10 \mathrm{eV}$ in its $v_{\mu}$ disappearance atmospheric neutrino sample are studied. The currently released 1-year data shows a mild (around $2 \sigma$ ) preference for non-zero sterile mixing, which overlaps with the favored region for the sterile neutrino interpretation of the ANITA upward shower. Although the null results from CHORUS and NOMAD on $v_{\mu}$ to $v_{\tau}$ oscillations in vacuum disfavor the hint from the IceCube 1-year data, the relevant oscillation channel and underlying physics are different. The physics reach of the roughly 8 years of data that is already on tape as well as a forecast of 20 years data to probe the present hint or improve upon current constraints are also discussed.
\end{abstract}

The 20th International Workshop on Neutrinos (NuFact2018)

12-18 August 2018

Blacksburg, Virginia

\footnotetext{
* Speaker.

${ }^{\dagger}$ This project has received funding/support from the European Union's Horizon 2020 research and innovation programme under the Marie Skłodowska-Curie grant agreement No 674896.
} 


\section{Introduction}

The simplest extension of the Standard Model (SM) of particle physics able to account for the evidence for neutrino masses and mixings consists in the addition of right-handed neutrinos to its particle content. The new physics scale is the Majorana mass of the new states and, since it is not related to the electroweak symmetry breaking mechanism, there is no theoretical guidance for its value. A large Majorana scale leads to the celebrated seesaw mechanism [1, 2, 3, 4], providing a very natural explanation of the lightness of neutrino masses. Conversely, a light neutrino mass could also naturally stem from a symmetry argument $[5,6,7,8,9,10]$. This proceeding is based on [11] where we studied the capabilities of the IceCube detector to look for sterile neutrinos in the average out regime by analyzing its atmospheric neutrino 1-year public data. For atmospheric neutrinos traveling through the Earth $(L \lesssim 12000 \mathrm{~km})$ and for the IceCube energies, the average-out limit happens for sterile neutrinos with masses above $10 \mathrm{eV}$.

\section{The neutrino oscillation probability at IceCube}

Following the parametrization introduced in [12], in presence of New Physics, the full leptonic mixing matrix that enters in the neutrino oscillation probability will be described by the PMNS mixing matrix and a lower triangular matrix $\alpha$ that encodes active-heavy mixing $U_{\alpha i}$.

The $v_{\mu}$ produced in the atmosphere from the collision of the cosmic rays with the nuclei of the atmosphere travel through the Earth, and eventually can reach the IceCube experiment where they are measured. Neglecting the electron neutrino oscillations, the analytic $v_{\mu} \rightarrow v_{\mu}$ survival probability in the averaged-out regime and for small sterile neutrino mixing will be given by

$$
P_{\mu \mu}=\left(1-\alpha_{\mu \mu}\right)^{4}\left(1-\sin ^{2}\left(2 \theta_{m}\right) \sin ^{2}\left(\frac{\Delta_{m} L}{2}\right)\right)+\sum_{i=4}^{n}\left|U_{\mu i}\right|^{4},
$$

where $\theta_{m}$ and $\Delta_{m}$ are the effective mixing and mass difference, respectively.

Given the large uncertainties in the atmospheric neutrino flux, we will allow a free normalization of the events in our analysis, and therefore no sensitivity to the normalization factor of Eq. (2.1) is expected. Since the leaking term does not depend on the energy nor the baseline, the last term of Eq. (2.1) can be dropped too. Thus, at leading order in $\alpha$, neglecting the atmospheric oscillation driven by $\Delta m_{31}^{2}$, and in the case of just one extra sterile neutrino Eq. (2.1) simplifies to

$$
P_{\mu \mu} \simeq 1-V_{\mathrm{NC}}^{2}\left|U_{\tau 4}\right|^{2}\left|U_{\mu 4}\right|^{2} L^{2},
$$

where $V_{\mathrm{NC}}$ is the matter potential from neutral current interactions.

\section{The analysis}

First, the atmospheric neutrino flux has been computed adopting the Honda Gaisser model [13] with the hadronic model QGSJET II-04 [14]. Then, the propagation of the neutrino flux trough the Earth has been simulated by using the nuSQuIDS software [15]. After propagating the flux for every value of the sterile neutrino parameter, the Monte Carlo provided with the data has been used to compute the expected number of events in every bin of reconstructed zenith angle. Notice that 
the observable $P_{\mu \mu}$ does not depend on the energy at leading order, and thus one energy bin and 40 bins of reconstructed zenith angle have been used in the analysis. Finally, the log-likelihood has been computed and minimized for a set of nuisance parameters in order to account for the uncertainties in the pion-kaon ratio of the initial atmospheric neutrino flux, the efficiency of the IceCube Digital Optical Modules, and the overall flux normalization.

\section{Results and conclusions}
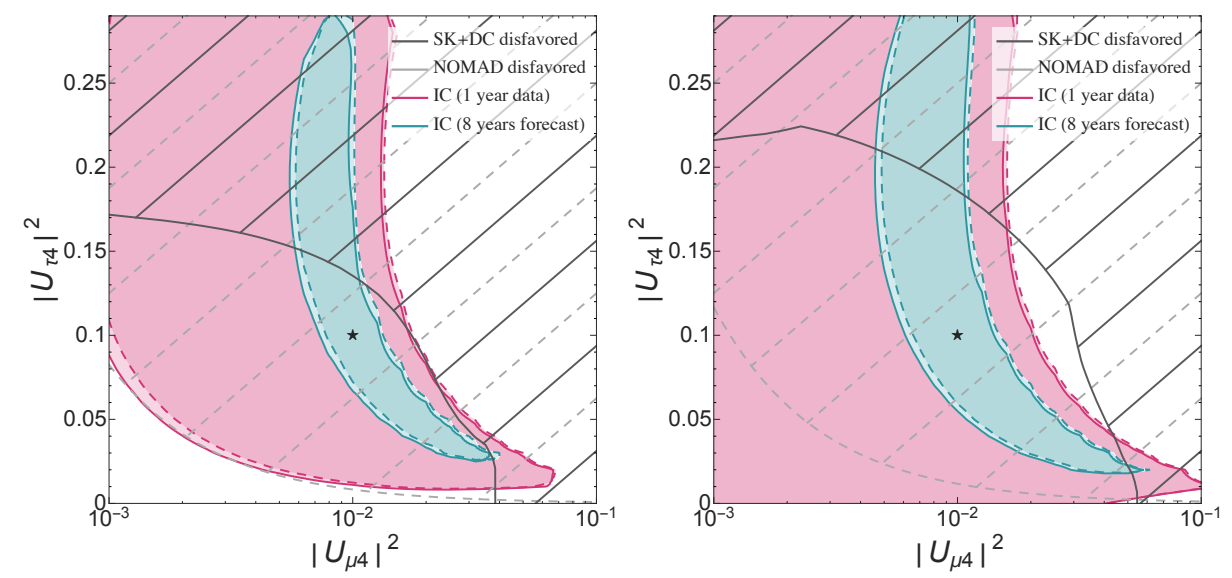

Figure 1: The left (right) panel shows in pink the constraints at 90\% (99\%) CL for the sterile mixing elements from the released 1-year data.

Left (right) panel of Figure 1 shows the obtained constrains from the IceCube experiment at $90 \%$ CL (99\% CL). In pink we show the constraints for the sterile mixing elements obtained from the 1-year released data. The cyan region shows at the same CL the forecast for 8 years of IceCube data assuming as true values the ones marked by the star. In particular, if the values of the sterile neutrino mixing marked by the star were realized in nature, 8-years of IceCube data would disfavor no sterile mixing around the $5 \sigma$ level. The solid hatched regions are already disfavored by SuperKamiokande [16] and DeepCore [17] experiments. At 99\% CL the obtained bounds improve over present SuperKamiokande and DeepCore constraints in some parts of the parameter space.

A mild preference for non-zero mixing with a significance of $2.3 \sigma$ is obtained in our analysis. However, we have observed that the significance of this preference ranges between 0.75 to $3 \sigma$ depending on the binning and atmospheric neutrino flux adopted.

Interestingly our results overlap with the favored region for the sterile neutrino interpretation [18] of the upward shower observed by ANITA [19]. However, the obtained preferred mixings are in tension with the null data of NOMAD [20] (dashed hatched regions of Figure 1). Nevertheless, the channel and underlying physics explored to obtain the bounds are very different in the two sets of experiments. While SuperKamiokande, DeepCore and IceCube analyze $v_{\mu}$ disappearance and the steriles are probed via their matter effects as shown in Eq. (2.2), NOMAD searched for $v_{\tau}$ appearance essentially in vacuum. Thus, in presence of non-standard matter effects the two results could still be reconciled. 


\section{References}

[1] P. Minkowski, $\mu \rightarrow$ e $\gamma$ at a Rate of One Out of $10^{9}$ Muon Decays?, Phys. Lett. 67B (1977) 421.

[2] R. N. Mohapatra and G. Senjanovic, Neutrino Mass and Spontaneous Parity Nonconservation, Phys. Rev. Lett. 44 (1980) 912.

[3] T. Yanagida, HORIZONTAL SYMMETRY AND MASSES OF NEUTRINOS, Conf. Proc. C7902131 (1979) 95.

[4] M. Gell-Mann, P. Ramond and R. Slansky, Complex Spinors and Unified Theories, Conf. Proc. C790927 (1979) 315 [1306.4669].

[5] R. N. Mohapatra and J. W. F. Valle, Neutrino Mass and Baryon Number Nonconservation in Superstring Models, Phys. Rev. D34 (1986) 1642.

[6] J. Bernabeu, A. Santamaria, J. Vidal, A. Mendez and J. W. F. Valle, Lepton Flavor Nonconservation at High-Energies in a Superstring Inspired Standard Model, Phys. Lett. B187 (1987) 303.

[7] G. C. Branco, W. Grimus and L. Lavoura, The Seesaw Mechanism in the Presence of a Conserved Lepton Number, Nucl. Phys. B312 (1989) 492.

[8] W. Buchmuller and D. Wyler, Dilatons and majorana neutrinos, Phys. Lett. B249 (1990) 458.

[9] A. Pilaftsis, Radiatively induced neutrino masses and large Higgs neutrino couplings in the standard model with Majorana fields, Z. Phys. C55 (1992) 275 [hep-ph/ 9901206 ].

[10] J. Kersten and A. Yu. Smirnov, Right-Handed Neutrinos at CERN LHC and the Mechanism of Neutrino Mass Generation, Phys. Rev. D76 (2007) 073005 [0 705.3221$].$

[11] M. Blennow, E. Fernandez-Martinez, J. Gehrlein, J. Hernandez-Garcia and J. Salvado, IceCube bounds on sterile neutrinos above $10 \mathrm{eV}$, Eur. Phys. J. C78 (2018) 807 [1803. 02362].

[12] M. Blennow, P. Coloma, E. Fernandez-Martinez, J. Hernandez-Garcia and J. Lopez-Pavon, Non-Unitarity, sterile neutrinos, and Non-Standard neutrino Interactions, JHEP 04 (2017) 153 [1609.08637].

[13] T. K. Gaisser, T. Stanev and S. Tilav, Cosmic Ray Energy Spectrum from Measurements of Air Showers, Front. Phys.(Beijing) 8 (2013) 748 [1303. 3565].

[14] S. Ostapchenko, Monte Carlo treatment of hadronic interactions in enhanced Pomeron scheme: I. QGSJET-II model, Phys. Rev. D83 (2011) 014018 [1010 . 1869].

[15] C. A. Argüelles Delgado, J. Salvado and C. N. Weaver, v-SQuIDS, .

[16] SUPER-KAMIOKANDE collaboration, K. Abe et al., Limits on sterile neutrino mixing using atmospheric neutrinos in Super-Kamiokande, Phys. Rev. D91 (2015) 052019 [1410.2008].

[17] ICECUBE collaboration, M. G. Aartsen et al., Search for sterile neutrino mixing using three years of IceCube DeepCore data, Phys. Rev. D95 (2017) 112002 [1702 . 05160].

[18] J. F. Cherry and I. M. Shoemaker, A Sterile Neutrino Origin for the Upward Directed Cosmic Ray Showers Detected by ANITA, 1802.01611.

[19] ANITA collaboration, P. W. Gorham et al., Characteristics of Four Upward-pointing Cosmic-ray-like Events Observed with ANITA, Phys. Rev. Lett. 117 (2016) 071101 [1603.05218].

[20] NOMAD collaboration, P. Astier et al., Final NOMAD results on muon-neutrino $\rightarrow$ tau-neutrino and electron-neutrino $\rightarrow$ tau-neutrino oscillations including a new search for tau-neutrino appearance using hadronic tau decays, Nucl. Phys. B611 (2001) 3 [hep-ex/0106102]. 